\title{
Kidney Diseases Enhance Expression of Tetraspanin-8: A Possible Protective Effect against Tubular Injury
}

\author{
Takashi Hirukawa $^{a}$ Qiong Wu ${ }^{a}$ Kaichiro Sawada ${ }^{a}$ Taiji Matsusaka ${ }^{a}$ \\ Sanae Saka ${ }^{c}$ Akira Oka $^{b}$ Nobuhito Hirawa ${ }^{c}$ Satoshi Umemura ${ }^{c}$

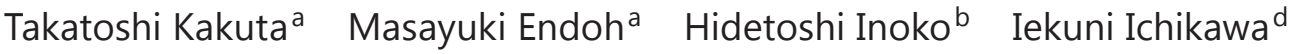 \\ Masafumi Fukagawa ${ }^{a}$ \\ a Division of Nephrology, Endocrinology and Metabolism, Department of Internal Medicine, \\ ${ }^{b}$ Department of Molecular Life Science and Molecular Medicine, Tokai University School \\ of Medicine, Isehara, and ${ }^{C}$ Department of Medical Science and Cardiorenal Medicine, \\ Yokohama City University, Graduate School of Medicine, Yokohama, Japan; d Division of \\ Pediatric Nephrology, Vanderbilt University School of Medicine, Nashville, Tenn., USA
}

\section{Key Words}

Immunoglobulin A nephropathy · Tetraspanin · Tubulointerstitial injury · Migration

\section{Abstract}

Background/Aims: TSPAN8 encoding tetraspanin-8 was identified as a candidate gene for immunoglobulin A nephropathy (IgAN) by a genome-wide association study using microsatellites in the Japanese population. Tetraspanin- 8 is a cell surface protein that contributes to the migration and invasion of epithelial cells. Methods: We performed immunohistochemistry for tetraspanin-8 on human renal biopsy specimens associated with IgAN, antineutrophil cytoplasmic antibody-associated nephropathy and interstitial nephritis, as well as normal renal tissue. Furthermore, to study the potential function of tetraspanin-8, we performed cell migration and invasion assays using human renal tubule cells transfected with tetraspanin-8. Results: Tetraspanin-8 was often expressed in vascular smooth muscle cells and occasionally in tubule cells in normal kidney. In the kidneys of all types of nephropathy, tetraspanin-8 staining in the arteries was unaffected, but that in the tubules was enhanced. The degree of tubular staining negatively correlated with the estimated glomerular filtration rate, independently of the type of nephropathy. Tetraspanin-8-expressing tubule cells were found predominantly in distal and collecting tubules, identified by cytokeratin 7 or aquaporin 2 staining. In vitro studies using cultured tubule cells revealed that tetraspanin-8 promoted migration by 2.7 -fold

Part of this study was presented at the 43rd Annual Meeting of the American Society of Nephrology, Renal Week 2010, Denver, Colo., USA, and published in the form of an abstract. 
Hirukawa et al.: Kidney Diseases Enhance Expression of Tetraspanin-8: A Possible Protective Effect against Tubular Injury

without laminin, by 2.8 -fold with laminin and invasion into Matrigel by 3.5 -fold, suggesting that enhanced tetraspanin-8 may be involved in the repair of tubules. Conclusion: The obtained findings indicate that tetraspanin-8 expression is enhanced in injured distal tubules, which may be involved in the repair of tubules by facilitating migration and invasion.

(C) 2014 S. Karger AG, Basel

\section{Introduction}

Immunoglobulin A nephropathy (IgAN) is the most common form of primary glomerulonephritis, causing end-stage renal disease that requires renal replacement therapy [1]. Ethnic variation in its prevalence and familial clustering suggest that IgAN is caused by a combination of genetic and environmental factors [2]. Previous genetic studies performed on various ethnic groups identified several susceptibility genes for IgAN [3-6]. We developed microsatellite markers covering the whole genome [7-10] and performed genome-wide association studies (GWAS) in the Japanese population (450 IgAN cases and 450 controls). We identified several susceptibility loci for IgAN in one of our previous studies [manuscript in preparation; some of the data were presented by Saka and colleagues at the American Society of Nephrology (ASN) meeting in 2009]. Among these loci, we focused on chromosome 12q21.1, which harbors microsatellites significantly associated with IgAN (D12S0177i: $\mathrm{p}=$ 0.001, AC001: $\mathrm{p}=8.71 \mathrm{E}-7$ ) and TSPAN8 encoding the tetraspanin-8 protein.

The tetraspanin family is a group of cell surface glycoproteins that contain four transmembrane domains and two conserved extracellular loops [11]. Tetraspanins interact and form complexes with a wide variety of proteins, including other members of the tetraspanin family, integrins, receptors and signaling molecules, forming the 'tetraspanin-web'. Through these interactions, they play important roles in fundamental cellular processes such as migration, proliferation and differentiation.

The expressed sequence tag profile suggests that tetraspanin-8 mRNA is widely expressed in various tissues, including in the digestive tract, connective tissue, bone, muscle and kidney [12]. Tetraspanin-8 has also been intensively studied in the field of cancer. It has been shown to be highly expressed in colorectal, hepatocellular and pancreatic carcinoma cells along with integrin family proteins. Several studies have suggested that tetraspanin-8 is also involved in the migration and invasion of cancer cells [13-16]. However, the detailed cellular expression pattern and function of tetraspanin-8 in human kidney have not been reported.

Regarding the interaction between tetraspanin and IgAN, Rops et al. [17] demonstrated that null-mutant mice for CD37, another member of the tetraspanin family, develop IgA glomerular disease similar to nephropathy, indicating that CD37 modulates the formation of IgA immune complexes. However, there have been no reports showing the relationship between TSPAN8 and IgAN.

We postulated that tetraspanin-8 may be differently expressed in chronic kidney diseases including IgA nephropathy. In the present study, we analyzed its expression pattern in human renal tissues from patients with several kidney diseases and explored its potential function using cultured human tubular epithelial cells (HK-2).

\section{Subjects and Methods}

Immunohistochemistry

Samples from renal biopsies performed in our hospital for diagnostic purposes were used in this study. The ethics committee of the Tokai University approved this study, and 
Hirukawa et al.: Kidney Diseases Enhance Expression of Tetraspanin-8: A Possible Protective Effect against Tubular Injury

Table 1. Clinical parameters of the patients whose renal tissue was analyzed in the present study

\begin{tabular}{lcccc}
\hline & Normal $(\mathrm{n}=5)$ & IgAN $(\mathrm{n}=20)$ & ANCA $(\mathrm{n}=5)$ & IN $(\mathrm{n}=5)$ \\
\hline Age, years & $27.8 \pm 7.5$ & $35.5 \pm 15.7$ & $64 \pm 5.8$ & $49.2 \pm 23.1$ \\
Serum albumin, g/dl & $4.5 \pm 0.2$ & $4.2 \pm 0.5$ & $3.3 \pm 0.7$ & $4.1 \pm 0.4$ \\
eGFR ${ }^{\text {a }}$ ml/min/1.73 m & $100.7 \pm 32.8$ & $78.3 \pm 34.5$ & $16.9 \pm 11.9$ & $38.8 \pm 24.4$ \\
Urinary protein, g/day & $0.3 \pm 0.3$ & $0.7 \pm 0.8$ & $1.3 \pm 0.8$ & $0.5 \pm 0.4$ \\
Blood pressure, mm Hg & $118.8 \pm 14.9 / 74.0 \pm 4.7$ & $129.8 \pm 16.4 / 77.5 \pm 12.6$ & $147.6 \pm 14.6 / 80.2 \pm 11.5$ & $111.8 \pm 16.6 / 70.0 \pm 6.5$ \\
\hline
\end{tabular}

Data were obtained at the time of biopsy. ${ }^{a}$ The eGFR was calculated by the following modified version of an equation for estimating the GFR of Japanese patients: $\mathrm{eGFR}=194 \times \mathrm{age}^{-0.287} \times \mathrm{sCr}^{-1.094} \times 0.739$ (if female), where sCr indicates serum creatinine.

Table 2. Clinical data of patients with DM nephropathy ( $\mathrm{n}=5)$

\begin{tabular}{lc}
\hline & DM nephropathy \\
\hline Age, years & $48 \pm 13.8$ \\
Serum albumin, g/dl & $3.4 \pm 1.3$ \\
eGFR ${ }^{a}, \mathrm{ml} / \mathrm{min} / 1.73 \mathrm{~m}^{2}$ & $3.0 \pm 20.4$ \\
Urinary protein, g/day & $140.0 \pm 24.0 / 80.0 \pm 17.2$ \\
Blood pressure, mm Hg & \\
\hline \multicolumn{2}{c}{$\begin{array}{l}\text { Data were obtained at the time of biopsy. } \\
\text { a The eGFR was calculated by the following modified version of an }\end{array}$} \\
equation for estimating the GFR of Japanese patients: eGFR = 194 $\times$ \\
age ${ }^{-0.287} \times \mathrm{sCr}^{-1.094} \times 0.739$ (if female), where sCr indicates serum creat- \\
inine.
\end{tabular}

informed consent was obtained for the use of remnant biopsy tissue for research purposes. The following disease samples were investigated: $\operatorname{IgAN}(n=20)$, antineutrophil cytoplasmic antibody-associated nephropathy (ANCA; $n=5$ ), interstitial nephritis (IN; $n=5$ ), diabetic nephropathy (DN; $n=5)$ and minor glomerular abnormalities $(n=5)$. The latter were taken from patients with mild proteinuria, who showed normal morphology by light microscopy, and were considered as normal controls. Clinical data corresponding to the renal samples are presented as mean and standard deviation (tables 1,2). Since the DN samples were obtained by needle biopsy and contained a limited number of tubules compared with the other samples obtained by open biopsy, the results and statistical analyses were conducted separately for the DN samples (table 2).

The biopsy samples were snap-frozen in acetone with dry ice and stored at $-80^{\circ} \mathrm{C}$. A total of 4- $\mu \mathrm{m}$-thick sections were mounted on silane-coated slides, fixed in acetone for $10 \mathrm{~min}$ at $4{ }^{\circ} \mathrm{C}$ and incubated in methanol containing $0.3 \% \mathrm{H}_{2} \mathrm{O}_{2}$ for $30 \mathrm{~min}$ at room temperature to eliminate endogenous peroxidase activity. The sections were incubated in blocking solution (Protein Block serum-free; Dako, Kyoto, Japan) for 20 min at room temperature and then in polyclonal rabbit anti-human TSPAN8 antibody (1:500 dilution, ab7007; Abcam, Tokyo, Japan), monoclonal mouse anti-human CD151 antibody (1:100 dilution, 11G5a; AbD Serotec, Oxford, UK) and monoclonal mouse anti-CD9 antibody (1:1,000 dilution, ab111020; Abcam) overnight at $4{ }^{\circ} \mathrm{C}$ in a humidified chamber. After washing in phosphate-buffered saline, the slides were incubated with mouse polyclonal anti-rabbit IgG antibody conjugated to horseradish peroxidase (Histofine simple stain MAX PO; Nichirei, Tokyo, Japan) for $30 \mathrm{~min}$ at room temperature. The signal was visualized with diaminobenzidine, and the slides were then counterstained with periodic acid-Schiff stains and Mayer's hematoxylin. 
Hirukawa et al.: Kidney Diseases Enhance Expression of Tetraspanin-8: A Possible Protective Effect against Tubular Injury

\section{Identification of Tubule Cell Types Expressing Tetraspanin-8}

To identify the types of tubule cells that express tetraspanin-8, we performed immunohistochemistry on five renal tissues associated with IgAN. Adjacent sections were further stained for cytokeratin 7 (a marker of distal and collecting tubules, 1:200 dilution; Dako), aquaporin 2 (a marker of collecting tubules, 1:500 dilution; Lifespan, Seattle, Wash., USA) or aquaporin 1 (a marker of proximal tubules, 1:200 dilution; Abcam).

\section{Construction of Tetraspanin-8 Expression Plasmid}

A DNA fragment containing the tetraspanin-8 coding sequence was generated by PCR using the cDNA from human colorectal cancer tissue, which highly expressed tetraspanin-8. PCR was carried out using KODplus reagent and primers, 5' -CCA GAG CAT ATT GCA GGA CA-3' and $5^{\prime}$-GGG GTT TGA CTG ACG ATA CG-3', under the following conditions: initial denaturation at $94^{\circ} \mathrm{C}$ for $2 \mathrm{~min}$, followed by 33 cycles consisting of denaturation at $98^{\circ} \mathrm{C}$ for $10 \mathrm{~s}$, annealing at $55^{\circ} \mathrm{C}$ for $30 \mathrm{~s}$ and extension at $68^{\circ} \mathrm{C}$ for $60 \mathrm{~s}$. The 826 -bp product was cloned into the pIRESpuro3 vector (Clontech, Mountain View, Calif., USA). After verification of the sequence, plasmid DNA was extracted using the Plasmid Mega Kit (Qiagen, Hilden, Germany).

\section{Immunohistochemistry on Mouse Tissues}

Frozen kidney sections from normal and NEP25 mice 2 weeks after the injection of LMB2 ( $2.5 \mathrm{ng} / \mathrm{g}$ body weight) were fixed in $4 \%$ paraformaldehyde and stained using a monoclonal rat anti-mouse tetraspanin-8 antibody (R\&D Systems, Minneapolis, Minn., USA). Severe injury was exhibited in kidney of NEP25 mice, with tubular dilatation and diffuse glomerulosclerosis [18]. The staining was visualized using Cy3 anti-rat IgG antibody.

\section{Plasmid Transfection into Renal Tubular Epithelial Cells}

HK-2 cells, derived from human tubular epithelial cells [19], were cultured in Dulbecco's modified Eagle's medium (DMEM; high glucose) containing $10 \%$ fetal bovine serum at $37^{\circ} \mathrm{C}$ in $5 \% \mathrm{CO}_{2}$. HK-2 cells grown to $80 \%$ confluency were transfected with tetraspanin-8 expression plasmid using the Amaxa Nucleofector System (Catalog No. VPI-1005; Lonza, Basel, Switzerland), in accordance with the manufacturer's protocol. Briefly, $1 \times 10^{6}$ cells were resuspended in $100 \mu \mathrm{l}$ of Nucleofector solution containing $4 \mu \mathrm{g}$ of tetraspanin-8 DNA, together with $2 \mu \mathrm{g}$ of pmaxGFP vector, a green fluorescent protein expression vector, and electroporated using program T-20 with the Lonza Nucleofector II device.

Starting the following day, the cells were selected with $0.5-2 \mu \mathrm{g} / \mathrm{ml}$ puromycin for 1 week. After trypsinization, cells expressing high levels of green fluorescent protein were individually harvested using fluorescence-activated cell sorting (FACSCalibur; BD Biosciences, Fukushima, Japan) and cultured in 96-well plates. Six clones of stably transfected cells, each derived from a single cell, were obtained. One clone, which expressed high levels of tetraspanin-8 mRNA, was used in the present study.

\section{In vitro Migration and Invasion Assay}

Control or tetraspanin-8-expressing cells $\left(2 \times 10^{5} /\right.$ well $)$ were suspended in DMEM without fetal bovine serum and seeded on polycarbonate Transwell filters $(8-\mu \mathrm{m}$ pore size; BD Biosciences). The outer wells were filled with DMEM containing 10\% fetal bovine serum. After incubation at $37^{\circ} \mathrm{C}$ for $36 \mathrm{~h}$, nonmigrating cells in the inner wells were removed using a polycarbonate scraper. The cells that had migrated to the opposite surface of the filter were stained with hematoxylin and microscopically observed at $\times 100$ magnification. Cell number was counted in ten visual fields. This experiment was repeated three times. The migration assay using laminin-coated filter (mouse laminin, $5 \mu \mathrm{g} / \mathrm{cm}^{2}$ ) and the invasion assay using Matrigel-coated filter (BD Biosciences) were similarly performed. 
Hirukawa et al.: Kidney Diseases Enhance Expression of Tetraspanin-8: A Possible Protective Effect against Tubular Injury

Fig. 1. Tetraspanin-8 in artery. Vascular smooth muscle cells were intensely stained for tetraspanin-8. Approximately $70 \%$ of crosssections of the interlobular artery contained cells with intense tetraspanin-8 immunostaining. This image shows a kidney associated with IgAN. Similar vascular staining was frequently observed in normal, ANCA and IN kidneys. Tissue sections were counter-stained with periodic acid-Schiff; original magnification $\times 200$.

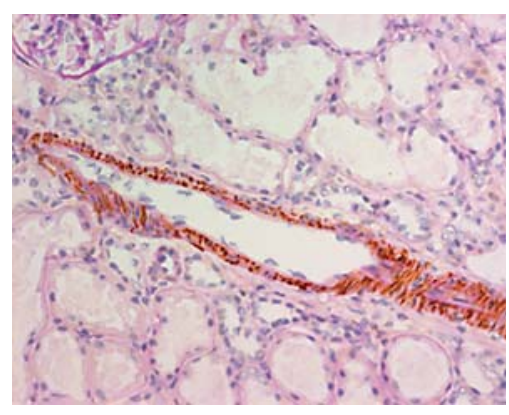

Fig. 2. Immunohistochemical detection of tetraspanin-8 in kidney associated with IgAN. Anti-tetraspanin-8 was stained in a fraction of tubule cells (arrows, left panel). The right panel shows the adjacent section stained without primary antibody. Tissue sections were counter-stained with periodic acid-Schiff; original magnification $\times 200$.

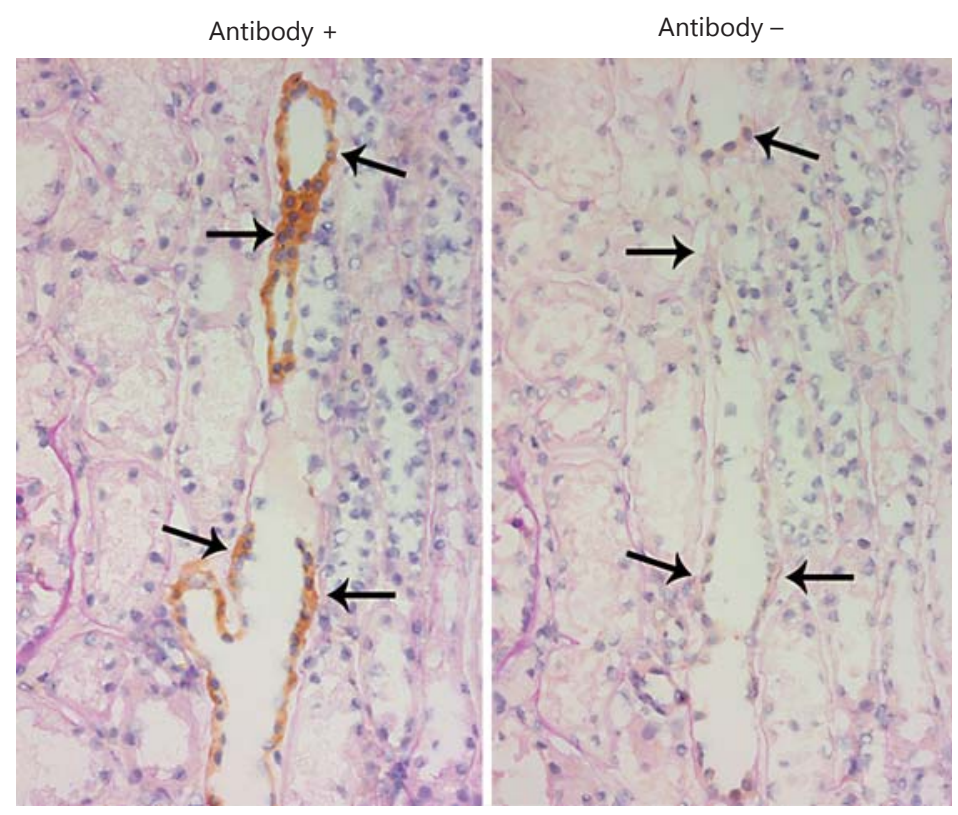

Fig. 3. Frequency of tetraspanin8-expressing tubules. Compared with normal kidneys, tetraspanin-8 expression was enhanced in kidneys associated with IgAN, ANCA, IN and DN. The horizontal bars represent medians.

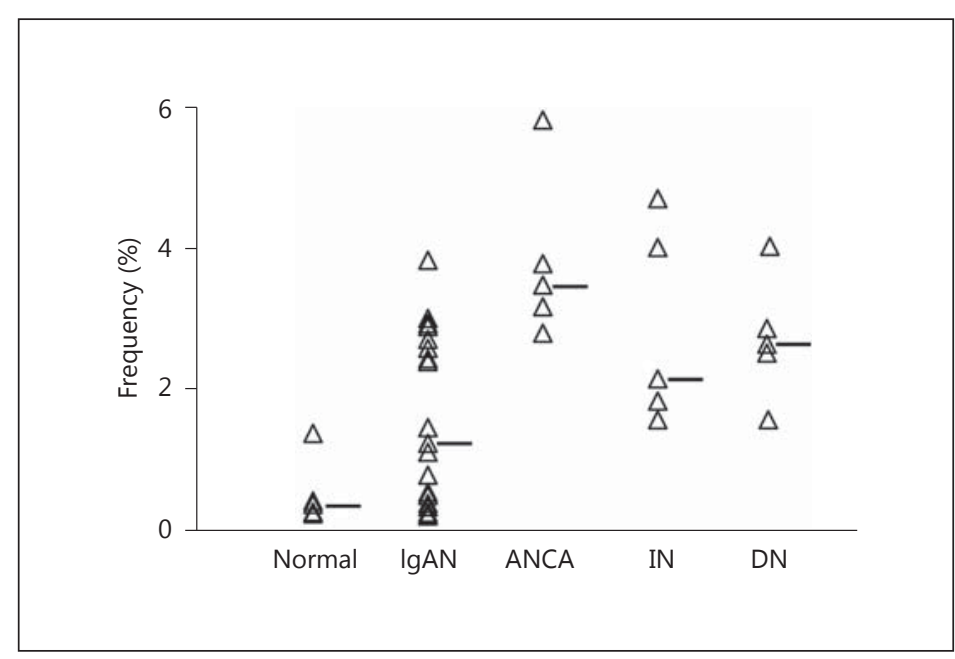


Hirukawa et al.: Kidney Diseases Enhance Expression of Tetraspanin-8: A Possible Protective Effect against Tubular Injury

\section{Statistical Analysis}

Clinical data corresponding to the renal samples are presented as mean \pm standard deviation (tables 1,2). Differences between two groups were analyzed by Student's t tests, and $p$ values $<0.05$ were considered statistically significant. The correlations between clinical parameters and tetraspanin-8 expression were evaluated by Spearman's nonparametric correlation coefficient analysis. Since DN samples were obtained by needle biopsy and contained a limited number of tubules compared with the other samples obtained by open biopsy, the correlations were analyzed separately for the DN samples and the rest. All analyses were performed using SPSS, version 15.0.

\section{Results}

\section{Expression of Tetraspanin-8 in Renal Tissues}

To elucidate the expression pattern of tetraspanin-8 in renal tissues, we performed immunohistochemical examination of renal biopsy samples. In normal renal tissue, tetraspanin-8 was often stained in vascular smooth muscle cells. Approximately $70 \%$ of cross-sections of interlobular arteries contained cells with intense tetraspanin-8 staining. The pattern and frequency of tetraspanin-8 staining in vascular smooth muscle cells were similar in tissues associated with IgAN, ANCA and IN (fig. 1).

In addition to vascular smooth muscle cells, tetraspanin-8 was occasionally stained in renal tubules of normal kidneys. Unlike staining in the arteries, tubular staining was enhanced in kidneys associated with IgAN, ANCA and IN (fig. 2). The median percentages of tubular cross-sections containing tetraspanin-8-positive cells were $0.34 \%$ in normal renal tissue, $1.15 \%$ in IgAN, $3.46 \%$ in ANCA, 2.13\% in IN and 2.35\% in DN (fig. 3). In renal tissue with severe tubulointerstitial injury, tubule cells more frequently expressed tetraspanin-8. However, within a given kidney, injured and intact tubules expressed tetraspanin-8 at similar frequencies.

We examined the correlation between tubular tetraspanin-8 positivity and various clinical factors at the time of biopsy in all samples obtained by open renal biopsy. Tubular tetraspanin-8 positivity was negatively correlated with estimated glomerular filtration rate (eGFR; $r=-0.730, p<0.001)$, and positively correlated with age $(r=0.768, p<0.001)$ and with the level of urinary protein $(r=0.537, p<0.001$; fig. $4 a-c)$. A similar negative correlation between tubular tetraspanin-8 and eGFR was also observed in kidney samples associated with DN ( $r=-0.900, p<0.01)$, which were obtained by needle biopsy (fig. 4 d).

We next determined the tubular segment expressing tetraspanin-8 by immunostaining in adjacent sections using antibodies for cytokeratin 7 (a marker of distal and collecting tubules), aquaporin 2 (a marker of collecting tubules) and aquaporin 1 (a marker of proximal tubules) (fig. 5). Of the tetraspanin-8-expressing tubule cells, $84.6 \%$ were also positive for cytokeratin 7 and $49.0 \%$ were positive for aquaporin 2 . In contrast, only $14.0 \%$ of tetraspanin8 -expressing cells were positive for aquaporin 1 . These observations indicate that tetraspanin-8 is predominantly expressed in distal and collecting tubules.

We compared the tetraspanin-8 expression pattern with those of two other tetraspanin families, CD151 and CD9. CD151 was intensely stained in glomeruli and smooth muscle cells, and weakly in renal tubules in normal kidney (fig. 6a). Basically, the same CD151 staining pattern was observed in renal tissues associated with all types of kidney disease (fig. 6b).

CD9 staining was intense in collecting tubules and vascular smooth muscle cells in normal kidney (fig. 6c, d). In kidney associated with ANCA and DN, CD9 staining in glomeruli was somewhat more intense than that in normal kidney (fig. 6e). The other types of kidney disease showed essentially the same pattern of CD9 staining. Tubular CD9 staining was similar in all types of kidney. 


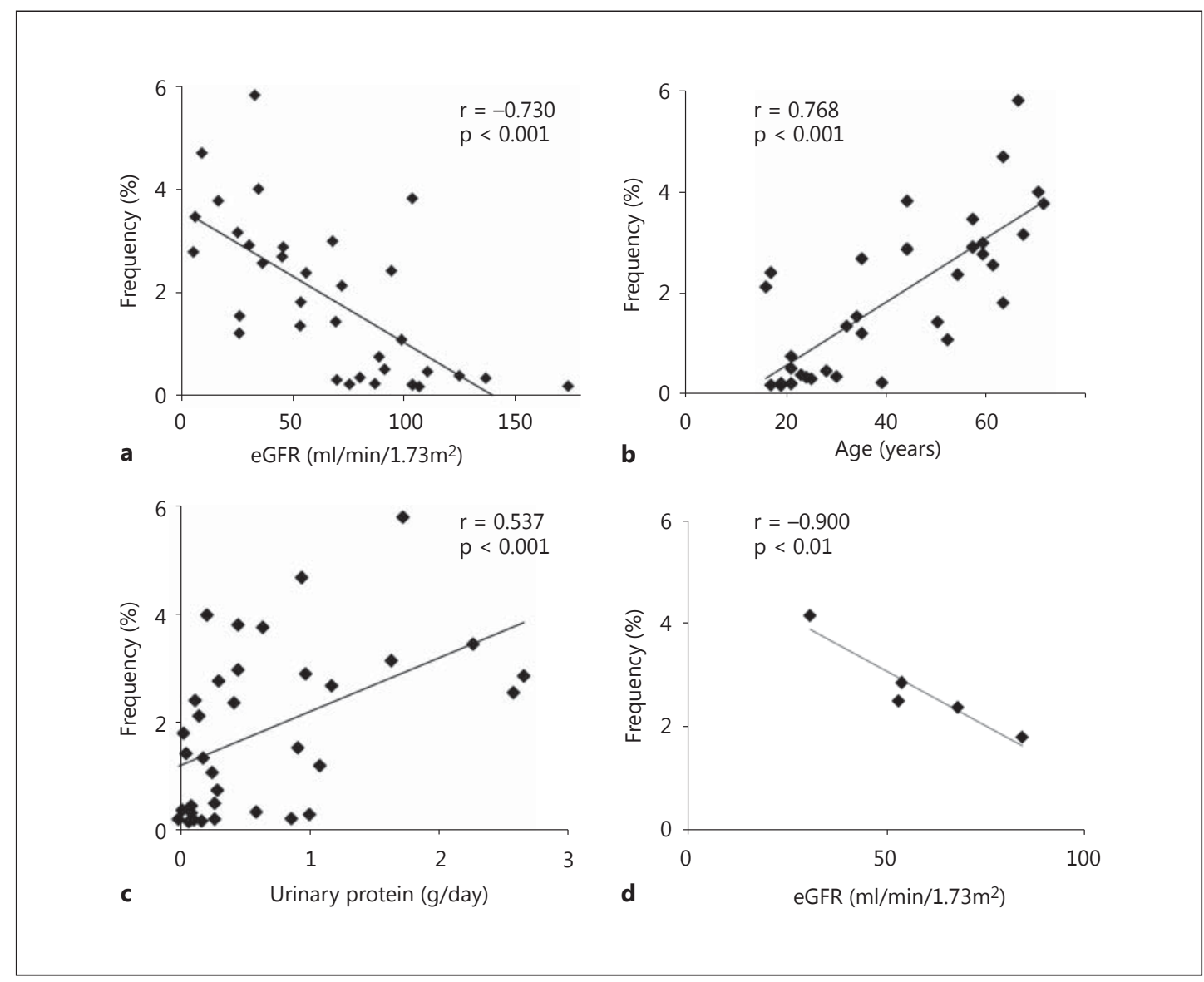

Fig. 4. Correlations of tetraspanin-8 expression and clinical parameters. When all biopsy samples were analyzed, the frequency of tetraspanin-8-expressing tubules showed a negative correlation with eGFR (a), and positive correlations with age (b) and urinary protein (c). The correlation of tubular tetraspanin-8 positivity and eGFR in DN is shown (d). The frequency of tetraspanin-8-expressing tubules showed a negative correlation with eGFR, as observed in other renal diseases.

Thus, the expression of CD9 overlapped with that of tetraspanin-8 in vascular smooth muscle cells, but the induced expression patterns were distinct in the tubules.

\section{Tetraspanin-8 Expression in Mouse Kidney}

We next examined tetraspanin-8 expression in mouse kidney. In normal kidney, tetraspanin-8 staining was observed in the distal and collecting tubules (fig. 7a). In kidney associated with severe podocyte injury, the staining was remarkably expanded and enhanced (fig. $7 b)$.

\section{Tetraspanin-8 Facilitates Tubule Cell Migration}

We next tested whether increased expression of tetraspanin-8 in renal tubule cells facilitates migration similarly to that in cancer cells $[13,14,20]$. For this, we performed a migration assay using the HK-2 cell line. When cells stably transfected with tetraspanin-8 expression plasmid were cultured on a filter with a pore size of $8 \mu \mathrm{m}$, an average of $22.3 \pm$ 9.2 cells in each visual field migrated across the filter within $36 \mathrm{~h}$. This was significantly more than the number of control cells $(8.2 \pm 4.8$; fig. $8 \mathrm{a})$. A similar result was obtained when the 
Tetraspanin-8

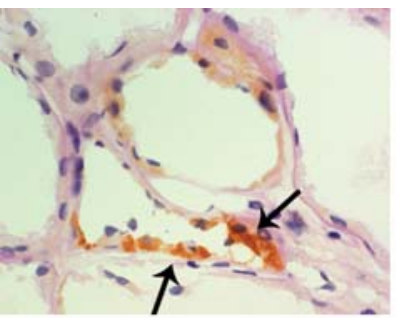

Cytokeratin 7

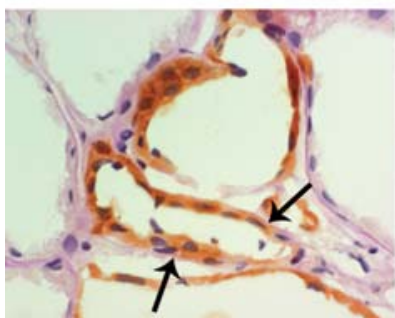

Tetraspanin-8

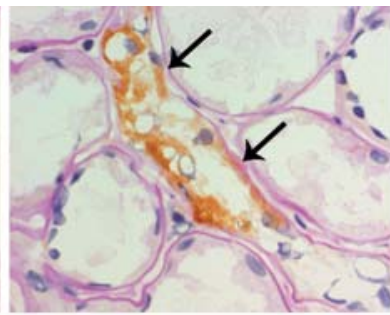

Aquaporin 2

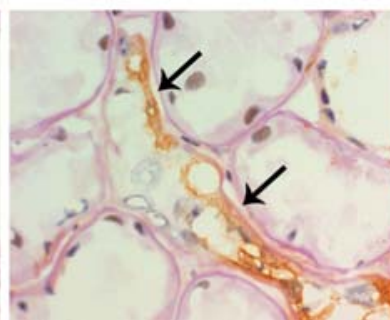

Tetraspanin-8

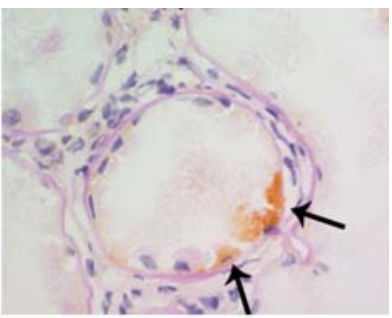

Aquaporin 1

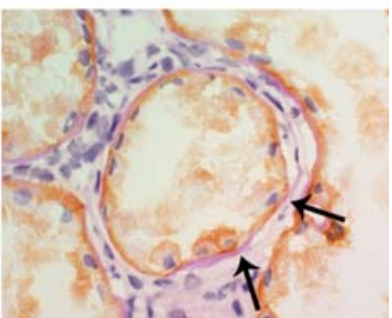

Fig. 5. Identification of tubule types expressing tetraspanin-8. Biopsy samples associated with IgAN were stained for tetraspanin-8 and the adjacent sections were stained for cytokeratin 7 (a marker of distal and collecting tubules), aquaporin 2 (a marker of collecting tubules) or aquaporin 1 (a marker of proximal tubules). Representative images are shown. The upper and lower panels show serial sections. In total, 84.6\% of tetraspanin-8-expressing cells were positive for cytokeratin 7, 49.0\% were positive for aquaporin 2 and $14.0 \%$ were positive for aquaporin 1 . Tissue sections were counter-stained with periodic acid-Schiff; original magnification $\times 400$.
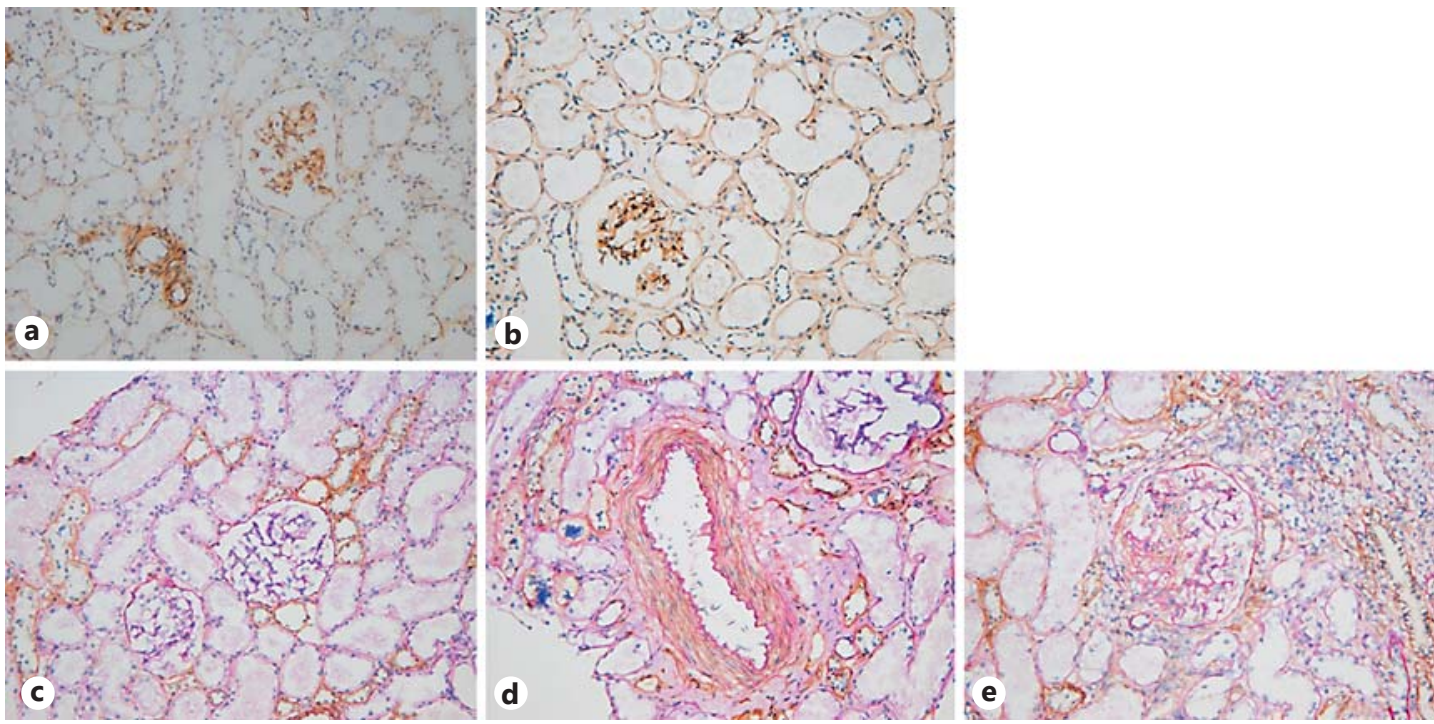

Fig. 6. Immunohistochemistry for CD151 and CD9 in kidney. a CD151 staining in normal kidney. CD151 was intensely stained in glomeruli and smooth muscle cells, and weakly in renal tubules. b CD151 in kidney with IgAN. The CD151 staining pattern was essentially the same as that of normal kidney. c, $\mathbf{d}$ CD9 staining in normal kidney. CD9 staining was intense in collecting tubules and vascular smooth muscle cells. e CD9 in kidney associated with ANCA. Injured glomerulus was faintly stained for CD9. The staining pattern in tubules was similar to that in normal kidney. Tissue sections were counter-stained with periodic acid-Schiff; original magnification $\times 400$. 

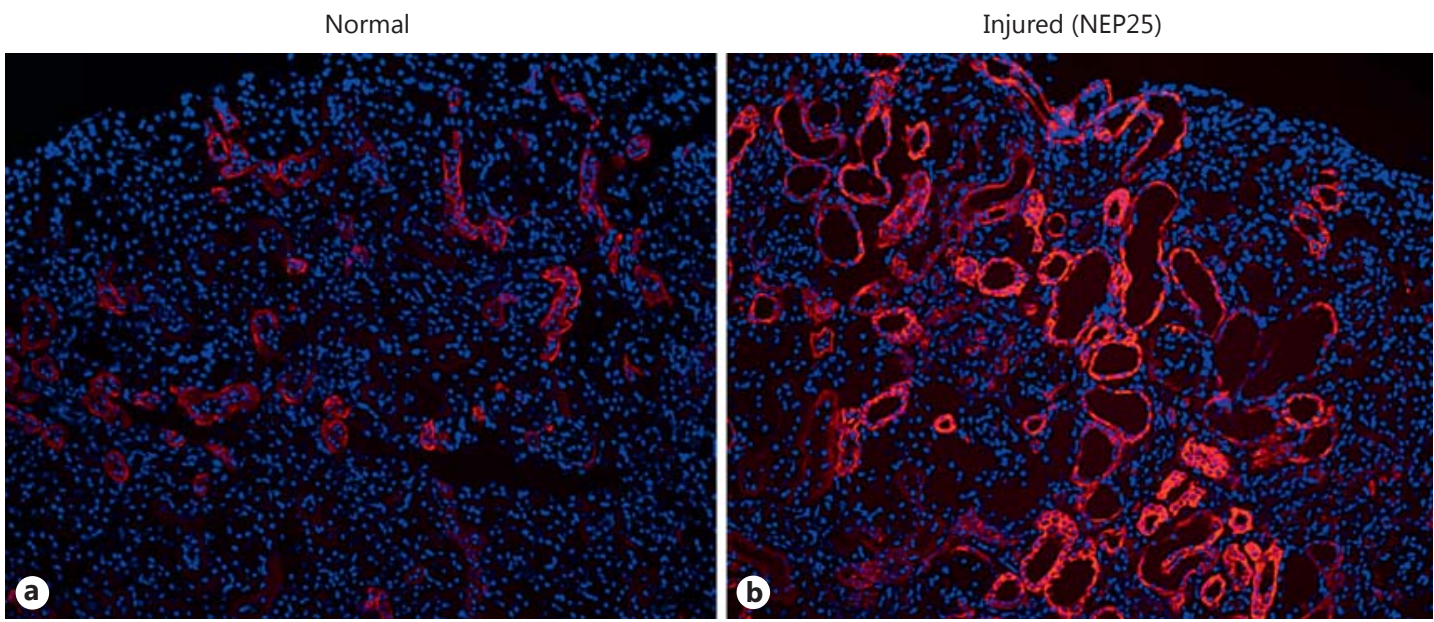

Fig. 7. Tetraspanin-8 in mouse kidney tissues. In normal kidney (a), tetraspanin-8 was observed in distal and collecting tubules. In kidney with severe podocyte injury (b), tetraspanin-8 expression was intensified and expanded.

Fig. 8. Tetraspanin-8 facilitates migration of tubule cells. HK-2 human tubule cells stably transfected with tetraspanin-8 expression plasmid (TSPAN8) and control cells (control) were cultured on a filter with a pore size of $8 \mu \mathrm{m}$ with (b) or without (a) laminin coating. Migrated cell numbers within $36 \mathrm{~h}$ were counted. Under both conditions, tetraspanin-8-expressing cells showed a significantly more active migration. ${ }^{*} \mathrm{p}<0.01$.

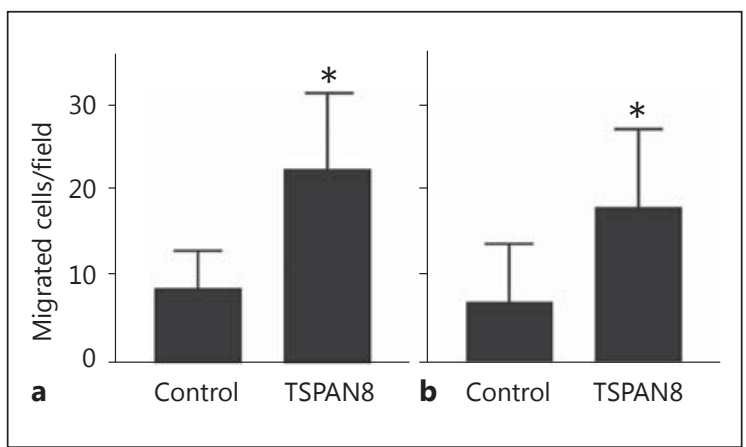

Fig. 9. Tetraspanin-8 facilitates invasion of tubule cells into the extracellular matrix. Invasion assay using Matrigel-coated filter showed that a greater number of tetraspanin-8-expressing tubule cells invaded and passed through the gel compared with the control HK-2 cells. ${ }^{*} \mathrm{p}<0.01$.

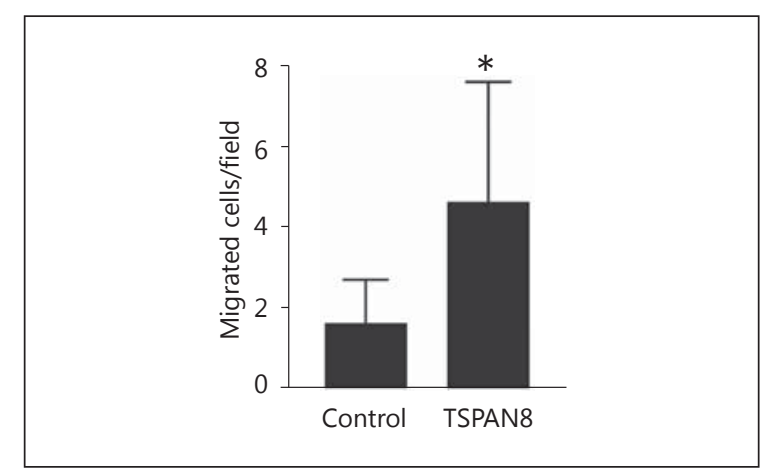

filters were coated with laminin. The average number of migrated tetraspanin-8-expressing cells was $17.8 \pm 9.2$, which was significantly more than that of control cells $(6.7 \pm 6.7$ per field; fig. 8b).

\section{Tetraspanin-8 Enhances Invasiveness of Tubular Cells}

It has been reported that tetraspanin-8 facilitates degradation of the extracellular matrix and the invasion of cancer cells into surrounding tissue [20]. We tested whether 
Hirukawa et al.: Kidney Diseases Enhance Expression of Tetraspanin-8: A Possible Protective Effect against Tubular Injury

tetraspanin-8 similarly enhances the invasiveness of tubule cells using a Matrigel-coated filter. On average, $1.3 \pm 1.3$ control cells were observed to invade the Matrigel layer and migrate across the filter in each visual field. In contrast, tetraspanin-8-expressing cells showed significantly increased invasiveness, with an average of $4.6 \pm 3.1$ migrated cells (fig. 9).

\section{Discussion}

Our immunohistochemical study revealed that tetraspanin-8 is expressed in some distal and collecting tubule cells and vascular smooth muscle cells. The frequency of tetraspanin-8expressing tubules was increased in damaged kidney, with a negative correlation with eGFR, but this finding was not specific to IgAN. These findings suggest that enhanced tubular expression of tetraspanin-8 is not a cause of IgAN, but rather reflects the severity of renal injury.

Of note, we originally identified TSPAN8 as a candidate susceptibility gene for the onset of IgAN through a GWAS comparing 450 biopsy-proven IgAN patients and 450 healthy subjects. A possible explanation of this unexpected result is that the IgAN cohort in our GWAS study may have included a significant number of patients with decreased renal function. At least, in most patients of this cohort the IgAN was severe enough to consider renal biopsy. Polymorphism in TSPAN8 may be associated with nonspecific progression of renal injury and accidentally chosen, although we have not studied the relationship between the risk alleles and tetraspanin-8 expression and function.

A similar pattern of tetraspanin-8 expression was also observed in mice. Thus, distal and collecting tubules express tetraspanin-8 in normal kidney. In kidney with severe podocyte injury, the staining was remarkably expanded and enhanced. The conserved expression pattern across species suggests that tetraspanin has an important role in kidney diseases. This induced expression in tubules was not observed for other tetraspanins, CD151 and CD9, suggesting that tetraspanin-8 has a unique role in kidney diseases.

The function of tetraspanin-8 in kidney has not been previously reported. In the field of cancer research, colorectal, hepatocellular and pancreatic carcinomas with higher tetraspanin-8 expression were shown to exhibit a higher frequency of invasion and metastasis than those with low expression, indicating that tetraspanin-8 regulates adhesion and motility via integrin and laminin [12-14, 16, 20]. In addition, Penas et al. [21] showed that tetraspanin modulates wound healing via adhesion and motility of keratinocytes. Our in vitro study showed that tetraspanin-8 facilitates migration and invasion of renal tubule cells. Therefore, it is speculated that tetraspanin-8 is involved in the process of repair of renal tubules, in which tubular cells acquire the capacity to move along the basement membrane to re-epithelialize wounded nephrons [22].

In our study, tetraspanin-8 staining did not always spatially coincide with tubular injury. This lack of local correlation indicates that tetraspanin-8 expression is not activated by a local mechanism, such as autocrine or paracrine secretion of TGF- $\beta$ [23]. Instead, it indicates that systemic factors that are evoked by, or closely correlated with, the decline of eGFR are involved in inducing tetraspanin-8 expression. Such regulation by systemic factors has been reported for other tetraspanins. For example, CD9 expression in renal tubules is enhanced by ischemia or hypertonicity $[24,25]$. We have not determined the systemic factors that enhance tetraspanin-8 expression, so this may be an interesting topic for future study. The candidates include uremic toxins and hypoxia. 


\section{Acknowledgements}

This work was supported by grants from the Tokai University, the Ministry of Health, Labor and Welfare and the Ministry of Education, Culture, Sports, Science and Technology of Japan. We thank the Education and Research Support Center of the Tokai University for technical assistance.

\section{Disclosure Statement}

The authors have no conflicts of interest to disclose.

\section{References}

1 Koyama A, Igarashi M, Kobayashi M: Natural history and risk factors for immunoglobulin A nephropathy in Japan. Research Group of Progressive Renal Disease. Am J Kidney Dis 1997;29:526-532.

-2 Barratt J, Feehally J: IgA nephropathy. J Am Soc Nephrol 2005;16:2088-2097.

-3 Gharavi AG, Yan Y, Scolari F, Schena PE, Frasca GM, Ghiggeri GM, Cooper K, Amoroso A, Viola BF, Battini G, Caridi G, Canova C, Farhi A, Subramnian V, Williams CN, Woodford S, Julian BA, Wyatt RJ, Lifton RP: IgA nephropathy, the most common cause of glomerulonephritis, is linked to 6q22-23. Nat Genet 2000;26:354357.

4 Bisceglia L, Cerullo G, Forabosco P, Torres DD, Scolari F, Perna MD, Foramitti M, Amoroso A, Bertok S, Floege J, Mertens PR, Zerres K, Alexopoulos E, Kirmizis D, Ermelinda M, Zelante L, Schena FP: Genetic heterogeneity in Italian families with IgA nephropathy: suggestive linkage for two novel IgA nephropathy loci. Am J Hum Genet 2006; 79:1130-1134.

5 Paterson AD, Liu XQ, Wang K, Magistroni R, Song X, Kappel J, Klassen J, Cattran D, George-Hyslop P, Pei Y: Genome-wide linkage scan of a large family with IgA nephropathy localizes a novel susceptibility locus to chromosome 2q36. J Am Soc Nephrol 2007;18:2408-2415.

6 Takei T, Iida A, Nitta K, Tanaka T, Ohnishi Y, Yamada R, Maeda S, Tsunoda T, Takeoka S, Ito K, Honda K, Uchida K, Tsuchiya K, Suzuki Y, Fujioka T, Ujiie T, Nagane Y, Miyano S, Narita I, Genjyo F, Nihei H, Nakamura Y: Association between single-nucleotide polymorphisms in selectin genes and immunoglobulin A nephropathy. Am J Hum Genet 2002;70:781-786.

7 Tamiya G, Shinya M, Imanishi T, Ikuta T, Makino S, Okamoto K, Furugaki K, Matsumoto T, Mano S, Ando S, Nozaki Y, Yukawa W, Nakashige R, Yamaguchi D, Ishibashi H, Yonekura M, Nakami Y, Takayama S, Endo T, Saruwatari T, Yagura M, Yoshikawa Y, Fujimoto K, Oka A, Chiku S, Linsen SE, Giphart MJ, Kulski JK, Fukazawa T, Hashimoto H, Kimura M, Hoshina Y, Suzuki Y, Hotta T, Mochida J, Minezaki T, Komai K, Shiozawa S, Taniguchi A, Yamanaka H, Kamatani N, Gojobori T, Bahram S, Inoko H: Whole genome association study of rheumatoid arthritis using 27,039 microsatellites. Hum Mol Genet 2005;14:2305-2321.

8 Yatsu K, Mizuki N, Hirawa N, Oka A, Ito N, Yamane T, Ogawa M, Shiwa T, Tabata Y, Ohno S, Soma M, Hata A, Nakano K, Ueshima H, Ogihara T, Tomoike H, Miki T, Kimura A, Mano S, Kulski JK, Umemura S, Inoko H: Highresolution mapping for essential hypertension using microsatellite markers. Hypertension 2007;49:446-452.

-9 Kawashima M, Tamiya G, Oka A, Hohjoh H, Juji T, Ebisawa T, Honda Y, Inoko H, Tokunaga K: Genomewide association analysis of human narcolepsy and a new resistance gene. Am J Hum Genet 2006;79:252-263.

10 Hui J, Oka A, James A, Palmer LJ, Musk AW, Beilby J, Inoko H: A genome-wide association scan for asthma in a general Australian population. Hum Genet 2008;123:297-306.

11 Helmer ME: Tetraspanin functions and associated microdomains. Nat Rev Mol Cell Biol 2005;6:801-811.

12 Champy MF, Le Voci L, Selloum M, Peterson LB, Cumiskey AM, Blom D: Reduced body weight in male Tspan8deficient mice. Int J Obes 2011;35:605-617.

13 Le Naour F, Abdre M, Greco C, Billard M, Sordat B, Emile JF, Lanza F, Boucheix C, Rubinstein E: Profiling of the tetraspanin web of human colon cancer cells. Mol Cell Proteomics 2006;5:845-847.

14 Sabine G, Paret C, Hildebrand D, Weitz J, Zgraggen K, Schmitz-Winnenthal FH, Horejsi V, Yoshie O, Herlyn D, Ashman LK, Zoller M: Colocalization of the tetraspanins, CO-029 and CD151, with integrins in human pancreatic adenocarcinoma: impact on cell motility. Clin Cancer Res 2005;11:2840-2852.

15 Lazo PA: Functional implications of tetraspanin proteins in cancer biology. Cancer Sci 2007;98:1666-1677.

16 Zoller M: Tetraspanins: push and pull in suppressing and promoting metastasis. Nat Rev Cancer 2009;9: 40-55.

17 Rops AL, Figdor CG, van der Schaaf A, Tamboer WP, Bakker MA, Berden JH, Dijkman HB, Steenbergen EJ, van der Vlag J, van Spriel AB. The tetraspanin CD37 protects against glomerular IgA deposition and renal pathology. Am J Pathol 2010;176:2188-2197. 
Matsusaka T, Xin J, Niwa S, Kobayashi K, Akatsuka A, Hashizume H, Wang Q, Pastan I, Fogo AB, Ichikawa I: Genetic engineering of glomerular sclerosis in the mouse via control of onset and severity of podocyte-specific injury. J Am Soc Nephrol 2005;16:1013-1023.

19 Ryan MJ, Johnson G, Kirk J, Fuerstenberg SM, Zager RA, Torok-Storb B: HK-2: an immortalized proximal tubule epithelial cell line from normal adult human kidney. Kidney Int 1994;45:48-57.

20 Zhou Z, Ran YL, Hu H, Pan J, Li ZF, Chen LZ, Sun LC, Peng L, Zhao XL, Yu L, Sun LX, Yang ZH: TM4SF3 promotes esophageal carcinoma metastasis via upregulating ADAM12m expression. Clin Exp Metastasis 2008;25:537548.

21 Penas PF, Garcia-Diez A, Sanchez-Madrid F, Yanez-Mo M: Tetraspanins are localized at motility-related structures and involved in normal human keratinocyte wound healing. J Invest Dermatol 2000;114:1126-1135.

-22 Zeisberg M, Maeshima Y, Mosterman B, Mosterman B, Kalluri R: Extracellular matrix microenvironment regulates migratory behavior of activated tubular epithelial cells. Am J Pathol 2002;160:2001-2008.

23 Lan HY, Chung AC: TGF- $\beta$ /Smad signaling in kidney disease. Semin Nephrol 2012;32:236-243.

-24 Takemura T, Hino S, Murata Y, Yanagida H, Okada M, Yoshioka K, Harris RC: Coexpression of CD9 augments the ability of membrane-bound heparin-binding epidermal growth factor-like growth factor (proHB-EGF) to preserve renal epithelial cell viability. Kidney Int 1999;55:71-81.

25 Sheikh-Hamad D, Ferraris JD, Dragolovich J, Preuss HG, Burg MB, García-Pérez A: CD9 antigen mRNA is induced by hypertonicity in two renal epithelial cell lines. Am J Physiol 1996;270:C253-C258. 